Original Research Article

\title{
Study of clinical and laboratory predictive markers of dengue fever and severe dengue in children
}

\author{
Prabhavathi R ${ }^{1}$, Madhusudan S R², Suman M.G ${ }^{3}$, Govindaraj $\mathbf{M}^{4}$, Puttaswamy $\mathbf{M}^{5}$, \\ ${ }^{1}$ Dr Prabhavathi R, Assistant Professor, ${ }^{2}$ Dr. Madhusudan S. R, Associate Professor, ${ }^{3}$ Dr. Suman M. G, Senior \\ Resident, ${ }^{4}$ Dr. Govindaraj M, Prof and HOD, All authors are affiliated with Department of Pediatrics, DR B. R. \\ Ambedkar Medical College, Bangalore, Karnataka, India, ${ }^{5} \mathrm{Dr}$ Puttaswamy M, Assistant Professor in \\ Biostatistics, Department of Community Medicine, Dr B.R Ambedkar Medical College, Bangalore, Karnataka, \\ India.
}

Address for Correspondence: Dr. Madhusudan S. R., 2231/4,16 ${ }^{\text {th }}$ A cross, $8^{\text {th }}$ main, D block, Sahakarnagar, Bengaluru-560092. Email-id: madhuravani28@gmail.com

\begin{abstract}
Introduction: Dengue fever is the most rapidly spreading arboviral infection with major public health consequences. Leucopenia, thrombocytopenia, hematocrit, elevated aminotransferases, low CRP and prolonged APTT, are useful predictive markers for early diagnosis of dengue infection. Objectives of the study: To study the clinical presentation and laboratory predictive markers of dengue fever, dengue with warning signs and severe dengue. Materials and Methods: Prospective hospital based study of 100 patients who were classified as dengue (DF), dengue fever with warning signs (DWS) and severe dengue (SD). Lab investigations included WBC count, Platelet count, Hematocrit, SGOT, SGPT, PT, APTT, INR, CRP. Clinical and laboratory parameters in the 3 groups of dengue fever were compared in the study. Results: Study group included 100 cases of which DF was 36\%, DWS 52\% and SD 12\%. Commonest presenting symptom was fever, nausea and vomiting. Pain abdomen, hepatomegaly, splenomegaly, bleeding, jaundice were seen in DWS and SD group. Lab parameters: Mean WBC count was low to normal in all 3 groups. Platelet count was low in all groups. Hematocrit, SGOT/SGPT, PT /APTT/INR was progressively found to be increased in the 3 groups. Low CRP was found in all 3 groups. Conclusion: Reliable diagnosis of dengue fever in endemic areas can be done by clinical parameters like presence of nausea, vomiting, pain abdomen and hepatomegaly. Ascites, pleural effusion, shock, liver dysfunction and hemorrhage are the markers for severe dengue. Monitoring platelet count, hematocrit and WBC count is very useful for management of dengue cases.
\end{abstract}

Keywords: Dengue fever, Leucopenia, Severe dengue, Thrombocytopenia, Warning signs

\section{Introduction}

Dengue is the most rapidly spreading mosquitoborne viral disease of mankind, with a 30 fold increase in global incidence over the last five decades. It is a major public health concern throughout the tropical and subtropical regions of the world [1]. According to WHO 2012 guidelines Dengue is classified into Dengue $+/$ - warning signs and severe dengue which includes Dengue shock syndrome, Respiratory distress syndrome, Dengue hemorrhagic fever and organ failure. In the febrile phase which lasts 2-7 days, patients typically

Manuscript received: $10^{\text {th }}$ May 2017

Reviewed: $21^{\text {st }}$ May 2017

Author Corrected: $30^{\text {th }}$ May 2017

Accepted for Publication: $7^{\text {th }}$ June 2017 develop a high-grade fever often accompanied by facial flushing, skin erythema, generalized body ache, myalgia, arthralgia, retro-orbital eye pain, photophobia, rubeliform exanthema and headache The warning signs mark the beginning of the critical phase.

Warning signs:

- Abdominal pain or tenderness

- Persistent vomiting

- Clinical fluid accumulation

- Mucosal bleed

- Lethargy; restlessness 
- Liver enlargement $>2 \mathrm{~cm}$

- Laboratory: Increase in HCT concurrent with rapid decrease in platelet count [2].

These patients become worse around the time of defervescence, when the temperature drops to $37.5-38^{\circ} \mathrm{C}$ or less and remains below this level, usually on days 3-8 of illness. Progressive leucopenia followed by a rapid decrease in platelet count usually precedes plasma leakage. An increasing haematocrit above the baseline may be one of the earliest additional signs. The period of clinically significant plasma leakage usually lasts 24-48 hours.

The degree of haemoconcentration above the baseline haematocrit reflects the severity of plasma leakage; however, this may be reduced by early intravenous fluid therapy. A right lateral decubitus chest radiograph, ultrasound detection of free fluid in the chest or abdomen, or gall bladder wall edema may precede clinical detection. In addition to the plasma leakage, hemorrhagic manifestations such as easy bruising and bleeding at venepuncture sites occur frequently [2].

Child with fever, nausea, vomiting, rash, aches and pain, positive tourniquet test is considered as probable dengue in endemic area and confirmed by NSI antigen / IGM antibody study. The lab findings of acute DF are as follows: Total WBC is usually normal at the onset of fever; then leucopenia develops with decreasing neutrophils. Platelet counts are initially normal. Mild thrombocytopenia (100 000 to 150000 cells $/ \mathrm{mm}^{3}$ ) is common and about half of all DF patients have platelet count below 100000 cells $/ \mathrm{mm} 3$, in severe dengue, platelet usually drops below $50000 / \mathrm{mm}^{3}$. Mild haematocrit rise $(\approx 10 \%)$ may be found. Serum biochemistry is usually normal but liver enzymes and aspartate aminotransferase (AST/SGOT) levels may be elevated mildly [1].

Clinical findings of Dengue Fever are: acute onset, high grade and continuous fever, nausea and/ vomiting and pain abdomen. Haemorrhagic manifestations including a positive tourniquet test, petechiae, purpura, ecchymosis, epistaxis, gum bleeding, and haematemesis and/or melena. Hepatomegaly is observed in $90 \%-98 \%$ of children. Shock is manifested by tachycardia, poor tissue perfusion with weak pulse and narrowed pulse pressure (20 $\mathrm{mmHg}$ or less) and hypotension as cold, clammy skin and/or restlessness. Lab features include: WBC count may be normal or with predominant neutrophils in the early febrile phase. Thereafter, there is a drop in the total number of white blood cells and neutrophils, reaching a nadir towards the end of the febrile phase. The change in total white cell count $(\leq 5000$ cells/mm3) and ratio of neutrophils to lymphocyte (neutrophils <lymphocytes) is useful to predict the critical period of plasma leakage. This finding precedes thrombocytopenia or rising haematocrit. A relative lymphocytosis with increased atypical lymphocytes is observed by the end of the febrile phase and into convalescence.

A sudden drop in platelet count to below 100000 occurs by the end of the febrile phase before the onset of shock or subsidence of fever. The level of platelet count is correlated with severity of DF. A sudden rise in haematocrit is observed simultaneously or shortly after the drop in platelet count. Haemoconcentration or rising haematocrit by $20 \%$ from the baseline is the objective evidence of plasma leakage.Thrombocytopenia, haemoconcentration and mildly elevated serum aspartate aminotransferase (AST/SGOT) levels $(\leq 200 \mathrm{U} / \mathrm{L})$ with the ratio of AST: ALT (SGOT/SGPT) $>2$ are constant findings in DF. Partial thromboplastin time and prothrombin time are prolonged in about half and one third of DF cases respectively.

Recovery phase: As the patient survives the 24-48 hour critical phase, a gradual reabsorption of extravascular compartment fluid takes place in the following 48-72 hours. General wellbeing improves, appetite returns, gastrointestinal symptoms abate, hemodynamic status stabilizes, and diuresis ensues. Generalized pruritus and bradycardia are common during this stage. The haematocrit stabilizes or may be lower due to the dilutional effect of reabsorbed fluid.

The white blood cell count usually starts to rise soon after defervescence but the recovery of the platelet count is typically later than that of the white blood cell count. Respiratory distress from massive pleural effusion and ascites, pulmonary edema or congestive heart failure will occur during the critical and/or recovery phases if excessive intravenous fluids have been administered [2]. Thrombin time is also prolonged in severe cases 
[1]. For a disease that is complex in its manifestations, management is relatively simple, inexpensive and very effective in saving lives, so long as correct and timely interventions are instituted [2]. The key to a good clinical outcome is understanding and being alert to the clinical problems that arise during the different phases of the disease, leading to a rational approach in case management [3]. Other laboratory tests may be requested to confirm case, as being used in research. These tests take several days to weeks for the results and are not used in routine practice. They are therefore used mainly for epidemiological purposes $[4,5]$.

The causes of mortality in dengue infection can be predicted from simple parameters which may be used to develop a future scoring system to predict and manage dengue infection severity early in the course of the illness [6]. Leucopenia, thrombocytopenia, elevated aminotransferases, low CRP and prolonged APTT, were useful predictive markers for early diagnosis of dengue infections [7]. In our setup, certain routine clinical and laboratory parameters have been proposed which can be used for predicting the severity of dengue infection.

Objectives of the study: To study the clinical presentation and laboratory predictive markers of dengue fever, dengue with warning signs and severe dengue.

\section{Methods}

Study design: A prospective study.

Setting: Hospital based study.

Inclusion criteria: Children below 18 years admitted to pediatric ward with acute onset high grade fever and Non structural antigen protein 1 [NS1] and/or Immunoglobulin M [IgM] positive.

Exclusion criteria: Children with other diseases like enteric fever, rickettsial fever, malaria, leptospirosis, septicemia and other viral hemorrhagic fevers.

Participants: Pediatric patients admitted to DR. B.R. Ambedkar Medical College.

Variables: Quantitative variables: Age, WBC count, Platelet count, hematocrit, Liver enzymes (SGOT\& SGPT), prothrombin time (PT), activated partial thromboplastin time (APTT), international normalized ratio (INR), $\mathrm{C}$ - reactive protein (CRP).

Qualitative variables: Fever, nausea, pain abdomen, hepatomegaly, splenomegaly, bleeding, pleural effusion, shock, jaundice, encephalopathy.

Data source: For data entry, questionnaire was used, where all the symptoms and lab investigations were entered and checked by senior consultants.

Bias: none

Study size: 100 patients.

Statistical methods: The results were analyzed using standard normal test and student ' $t$ ' test.

After clinical assessment, the patients were classified as dengue, dengue fever with warning signs and severe dengue. Lab investigations included $\mathrm{CBC}$, especially $\mathrm{WBC}$ count, platelet count, hematocrit, SGOT, SGPT, PT, APTT, INR, and CRP was monitored .Serial monitoring for clinical and hematological parameters were done. Cut-off values for laboratory tests are defined as followings: leucopenia (white blood cell count < $4000 / \mathrm{mm}^{3}$ ), thrombocytopenia (platelet count $<$ $150 \times 103 / \mathrm{mm}^{3}$ ) prolonged activated partial thromboplastin time (APTT) ( $>38 \mathrm{sec}$ ), elevated serum aminotransferase levels (aspartate aminotransferase (AST) or alanine aminotransferase $(\mathrm{ALT})>39 \mathrm{U} / \mathrm{L})$ and low C-reactive protein (CRP) $(<20 \mathrm{mg} / \mathrm{L})$. Clinical and laboratory parameters in dengue fever, dengue fever with warning signs and severe dengue were compared in the study.

\section{Results}

Study group included 100 cases of which DF was 36\%, DWS 52\% and SD 12\%. Commonest presenting symptom was fever followed by nausea and vomiting ( $\mathrm{DF}=50 \%, \mathrm{DWS}=84.6 \%$ \& $\mathrm{SD}=83.3 \%$ ). Pain abdomen (DWS $=80.8 \%, S D=75 \%$ ), hepatomegaly $(\mathrm{DF}=11.1 \%, \mathrm{DWS}=46.2 \%, \mathrm{SD}=75 \%)$, splenomegaly $(\mathrm{DWS}=26.9 \%$, $\mathrm{SD}=25 \%$ ), bleeding (DWS $=40.4 \%, \mathrm{DWS}=34.6 \%$ and $\mathrm{SD}=66.7 \%$ group). Shock was observed in $75 \%$ of $\mathrm{SD}$ patients. Jaundice was seen in $3.8 \%$ of DWS and $25 \%$ in SD group. 2 patients in SD had dengue encephalopathy. 
Lab parameters included, Mean WBC count in DF was 4866.7, DWS-5380.3, SD-8429.2. Mean Platelet count was 1.31 lakh in DF, 1.02 lakh in DWS and 0.31 lakh in SD groups. Mean Hematocrit was 33.8, 34.4 and 40.3 in DF, DWS and SD group respectively. The mean SGOT/SGPT in DF was 93.4 and 53.3, in DWS was 106.2 and 65.3, and in SD 238.1 and 193.8 respectively.PT /APTT/INR was progressively found to be increased in the 3 groups. There was no significant difference in CRP in the 3 groups. Results have been tabulated in Table 2.

Table-1: Clinical signs and symptoms.

\begin{tabular}{|c|c|c|c|c|}
\hline \multirow{2}{*}{ Parameter } & $\mathbf{D F}(\mathbf{N}=\mathbf{3 6})$ & $\mathbf{D W S}(\mathbf{N}=\mathbf{5 2})$ & $\mathbf{S D}(\mathbf{N}=\mathbf{1 2})$ & $\begin{array}{c}\text { P-value (DWS vs. } \\
\text { SD) }\end{array}$ \\
\cline { 2 - 4 } & $\mathbf{N}(\mathbf{\%})$ & $\mathbf{N}(\mathbf{\%})$ & $\mathbf{N}(\mathbf{\%})$ & NA \\
\hline Fever & $36(100)$ & $52(100)$ & $12(100)$ & 0.9122 \\
\hline Nausea & $18(50)$ & $44(84.6)$ & $9(83.3)$ & 0.6543 \\
\hline Pain abdomen & $0(0)$ & $42(80.8)$ & $9(75)$ & 0.0715 \\
\hline Hepatomegaly & $4(11.1)$ & $24(46.2)$ & $3(25)$ & 0.8919 \\
\hline Splenomegaly & $0(0)$ & $14(26.9)$ & $4(33.3)$ & 0.6518 \\
\hline Bleeding & $0(0)$ & $21(40.4)$ & $8(66.7)$ & $0.0002^{*}$ \\
\hline Pleural effusion & $0(0)$ & $8(15.4)$ & $8(66.7)$ & $0.0416^{*}$ \\
\hline Ascites & $0(0)$ & $18(34.6)$ & $9(75)$ & NA \\
\hline Shock & $0(0)$ & $0(0)$ & $3(25)$ & $0.0138^{*}$ \\
\hline Jaundice & $0(0)$ & $2(3.8)$ & $2(16.7)$ & NA \\
\hline Encephalopathy & $0(0)$ & $0(0)$ & & \\
\hline
\end{tabular}

$* \mathrm{P}<0.05$ is statistically significant; NA- not applicable

Table-2: Laboratory parameters.

\begin{tabular}{|c|c|c|c|c|}
\hline \multirow{2}{*}{ Parameters } & DF(N=36) & DWS(N=52) & SD(N=12) & $\begin{array}{c}\text { P-value (DWS } \\
\text { vs. SD) }\end{array}$ \\
\cline { 2 - 5 } & Mean \pm SD & Mean \pm SD & Mean \pm SD & 0.319 \\
\hline Age(years) & $8.6 \pm 4.4$ & $9.5 \pm 3.9$ & $8.3 \pm 3.4$ & $0.016^{*}$ \\
\hline WBC(cells/c.mm) & $4866.7 \pm 2458.7$ & $5380.6 \pm 3698.6$ & $8429.2 \pm 4518.9$ & $0.001^{*}$ \\
\hline $\begin{array}{c}\text { Platelet COUNT } \\
\text { (per c.mm) }\end{array}$ & $\begin{array}{c}130694.4 \pm \\
62581.1\end{array}$ & $102047.1 \pm 66213.1$ & $31250 \pm 9799.1$ & $0.024^{*}$ \\
\hline HCT (volume \%) & $33.8 \pm 4.1$ & $34.4 \pm 8$ & $40.3 \pm 7.3$ & $0.0001^{*}$ \\
\hline SGOT(AST)(IU/L) & $94.1 \pm 70.1$ & $106.2 \pm 51$ & $238.1 \pm 118.3$ & $0.0001^{*}$ \\
\hline SGPT(ALT)(IU/L) & $53.3 \pm 26.2$ & $65.3 \pm 37.4$ & $193.8 \pm 100.1$ & $0.003^{*}$ \\
\hline PT(seconds) & $12.4 \pm 1.1$ & $13.9 \pm 3.3$ & $17.3 \pm 3.5$ & $0.005^{*}$ \\
\hline APTT(seconds) & $26.7 \pm 4.1$ & $34.5 \pm 5.7$ & $39.9 \pm 6.2$ & $0.002^{*}$ \\
\hline INR & $1.1 \pm 0.3$ & $1.2 \pm 0.3$ & $1.4 \pm 0.3$ & 0.396 \\
\hline CRP(mg/dl) & $5.5 \pm 2.5$ & $4.4 \pm 3.1$ & $5.3 \pm 3.6$ & \\
\hline
\end{tabular}

$* \mathrm{P}<0.05$ is statistically significant

\section{Discussion}

Dengue virus was isolated in India for the first time in 1945. The first dengue hemorrhagic fever (DHF) outbreak occurred in Calcutta (West Bengal) in 1963. The agents of dengue, i.e. dengue viruses, are categorized under the genus Flavivirus. These viruses contain single stranded RNA and are small in size $(50 \mathrm{~nm})$. There are four dengue virus serotypes which are designated as DENV-1, DENV-2, DENV-3 and DENV-4.They can cocirculate in the endemic areas because the 
immunity to one serotype does not afford protection from the infection by a heterotopous serotype. Secondary infections are associated with elevated risks of severe disease outcomes [8].

Dengue viruses are transmitted from an infected person to others by the bite of the female mosquito. In India, Aedes aegypti is the main vector in most urban areas. Climatic conditions, particularly temperature and rainfall, have a major impact on the lifecycle, breeding and longevity of vectors and thus transmission of the disease. [8]. In our study, 100 cases of dengue fever were included from Nov 2015-Oct 2016 of which 48 were female and 52 were male. We found that maximum number of cases $(65 \%)$ were in the period from May 2016-Sep 2016 which coincides with the rainy season.

Fever, the most common symptom was present in all our patients. Nausea and vomiting was observed in $72 \%$ of our patients, more in the DWS group $(86.4 \%)$ and SD (83.3\%), comparable to other studies [9]. Saraswathy et al and Siddhart Bhave et al have reported vomiting in $50-79 \%$ of cases of DF and DHF [10,11]. Abdominal pain was reported in $80.8 \%$ in DWS group and $75 \%$ in SD groups. Surangrat et al observed that abdominal pain was present in $61.2 \%$ of cases with DHF and $66.7 \%$ cases in DSS [6]. Bokade et al also has observed abdominal pain in $25 \%$ of cases in DWS and $70 \%$ cases of SD [12]. Dhrubajyoti et al has noted similar findings [13]. Host immune responses play an important role in the pathogenesis of Dengue Fever (DF). The exact pathogenetic mechanism for different clinical manifestations of dengue fever is still not clearly understood. The most favored is virus strains enhancing antibodies and memory $\mathrm{T}$ cells in a secondary infection resulting in "Cytokine Tsunami". Other mechanism is demonstration of a shift from Th1-response in mild dengue to Th2-response in severe DHF. These ultimately target vascular endothelium, platelets and various organs leading to vasculopathy and coagulopathy responsible for the development of hemorrhage and shock. (Figure1) $[8,14]$.

In our study, bleeding, hepatomegaly and splenomegaly were observed in DWS and SD group. Bleeding was mainly in the form of epistaxis and melena. In dengue infection, due to thrombocytopenia and major hepatic dysfunction (raised SGPT, INR >1.5), there is decreased production of major plasminogen activator inhibitor-1 and excess activation of fibrinolytic process, that may lead to a tendency of spontaneous bleeding [15]. Abnormal coagulogram, platelet dysfunction, prothombin complex deficiency secondary to liver involvement, sequestration of platelets are also some of the mechanisms proposed for causes of bleeding. Liver involvement is found more frequently in infants compared to children [8]. Hemant Jain et al has reported epistaxis as the commonest type of bleeding whereas Baskar et al has showed that incidence of bleeding was 34\%, more in the DSS group [5,9]. Dhrubajyoti et al has found that bleeding is present in $46 \%$ of cases with DWS and $36 \%$ of cases with SD [13]. In another study by Siddhart Bhave et al, hematemesis was the major manifestation of bleeding [11].

Plasma leakage in dengue can lead to pleural effusion, ascites and shock. It usually becomes evident on 3 to 7 day of illness and patients may be afebrile during this time [8,16-19]. Ultrasonography is a valuable tool to detect fluid accumulation.Regular monitoring of blood pressure clinically helps to detect narrow pulse pressure and early onset of shock. Ascites and pleural effusion were present in DWS (34.6\%) and SD (66.7\%) group which was statistically significant $(\mathrm{p}<0.042$ and $\mathrm{p}<0.0002$ respectively).

Hemant Jain et al has reported that ascites is present in $15 \%$ of DWS and $8 \%$ of SD cases, whereas in the study by Tamil Selvan et al, ascites was found only in SD group [9, 20]. Baskar et al and Surangrat et al have reported that pleural effusion and ascites are present more in DWS and SD groups [5, 6]. Therefore, third space fluid accumulation can be considered as a sensitive clinical predictive marker of severity of dengue fever, including shock. Abdominal and thoracic sonography can be used as a first-line imaging modality in patients with suspected dengue fever to detect early signs suggestive of the disease [21]. The patients in our study with shock were managed conservatively according to WHO PROTOCOL (Fig 2). 5 patients had jaundice (2 in DWS and 3 in SD group) which was evaluated extensively for other causes and co-morbid conditions like malaria, enteric fever and viral hepatitis and were found to be negative. Liver enzymes were raised in all cases, more in DWS and SD groups which reflected the severity of the disease and were statistically 
significant $(p=0.0001)$. The degree of liver dysfunction in children with dengue infection varies from mild injury with elevation of transaminases to severe injury with jaundice and liver cell failure. In dengue, the rise of AST is usually more than ALT. By follow-up, AST levels return to normal levels in most of the cases. On the other hand ALT levels remain slightly increased above the normal cut-off value in approximately one-third of the patients.

This pattern, with AST rising more quickly and peaking at a higher level and then returning to normal faster than ALT levels, is different from the pattern usually seen in acute hepatitis caused by hepatitis viruses [13,22]. Our study revealed that AST was more than ALT in all the groups but we could not follow up adequate number of cases.

Prominence of musculoskeletal symptoms in dengue and skeletal muscle injury could explain the higher AST levels [12]. Dhrubajyoti has revealed that the liver enzymes were elevated AST - 84.6\% and $100 \%$ of DHF and DSS cases respectively and ALT was raised in $92 \%$ of DHF and $96 \%$ of DSS cases [13]. Tamil Selvan et al has mentioned that mean AST in 299 in SD group and 252 in DWS group. Mean ALT was 313 in SD group compared to 124 in DWS group [23]. CNS manifestation in dengue can present as encephalopathy, encephalitis, febrile seizures and intracranial bleed [8]. In our study, 2 patients had encephalopathy in the SD group which improved with conservative management in 2-3 days. There was no evidence of CNS bleeding in our study.

Leucopenia is a sensitive indicator for the diagnosis of dengue cases. In our study we found that WBC count was low in the DF group (mean=4866). In the DWS and SD group leukocyte counts were more (mean=5380 and $\mathrm{m}=8429$ ). Other studies have reported similar findings [6]. In one study by Hemant Jain et al, total count was less than 4000 in $55 \%$ of cases [9]. In the study by Saha et al, the majority of patients had leukocyte count between $3000-4000[19]$

Dhrubajyoti et al has reported uniformly low total count in all the 3 groups [17]. Thus leucocyte counts are an important guide for the initial diagnosis of dengue but normal WBC count can be found in severe cases, probably due to stress.
The platelet count was low in all the groups of dengue fever. It was significantly low (mean $=31250)$ in SD. The difference in platelet count between DWS stage (<1.02 lakh) and SD (31250) was significant $(\mathrm{p}<0.01)$ to classify the stage and severity of dengue and administer treatment accordingly. Hemant Jain et al reports thrombocytopenia $<1$ lakh in $80 \%$ of cases and $10 \%$ of patients had platelet count $<20000(10)$. Saraswathy et al has shown that platelet count was low in all cases of DHF and $44 \%$ in DF [10].

Siddhart Bhave et al has reported thrombocytopenia in $96 \%$ of dengue cases [11]. Dhrubajyoti et al observed that platelet count was low $(64.4 \%)$ in DF and all cases of DHF and DSS cases $(100 \%)$ [13]. The mean platelet count in SD group (31250) was comparable to other studies $[5,13]$. Low platelet count by itself does not predict the occurrence of bleeding. It is dependent on status of liver function and coagulation parameters. In our study, bleeding was present in $40.4 \%$ in DWS group and $33.3 \%$ in SD group. In a study from Taiwan, Ho et al has found that the combination of leucopenia $(<4000 / \mathrm{c} . \mathrm{mm})$, thrombocytopenia $\left(<150 \times 10^{3} / \mathrm{c} . \mathrm{mm}\right)$, prolonged APTT (>38s), elevated liver enzymes (AST/ALT $>1.5)$ and low CRP $(<20 \mathrm{mg} / \mathrm{dl})$ has a positive predictive value of $93.1 \%$ for early diagnosis of dengue [7].

In our study also, we have found that thrombocytopenia, elevated liver enzymes and abnormal coagulation profile are sensitive markers of DWS and SD cases and when considered together, can also predict the probability of bleeding. Ho et al also has shown that low CRP $(<20 \mathrm{mg} / \mathrm{dl})$, when taken alone has a positive predictive value of $81.7 \%$ and sensitivity of $93.8 \%$ for diagnosis of dengue [7]. Our study also revealed uniformly low CRP in all the patients, which helps to diagnose dengue and differentiates it from other conditions like bacterial infection, malaria, inflammatory diseases and malignancy.

Raised hematocrit was found more in DWS and SD group which was an important predictor of severity $(p=0.024)$. Baskar et al has reported mean hematocrit of 36.16 in DF and 36.69 in DSS, whereas in DHF it is 39.84 [5]. Siddhart Bhave et al has observed increased hematocrit in all the patients [11]. Saha et al has shown that hematocrit 
of $>35 \%$ has $95 \%$ specificity in predicting dengue (15). In our study, the values of PT, APTT and INR were progressively increased in all the 3 groups, which are markers for bleeding. The mean PT was 17.3 in SD group and mean INR 1.4. Mean APTT was 34.5 in DWS group and 39.9 in SD group. All these values were significant.

Surangrat et al has stated that mean PT was 15.5 and APTT was 44.55 in DSS group [6]. Tamil Selvan et al in 2015 has reported that INR $>1.5$ was elevated only in $3.3 \%$ of cases of SD. Similar study from the same author has shown this value has increased to $14.9 \%$ in a recent study [20,23]. This may show the worsening status of severe dengue infections over time. Kalenahalli et al also has shown INR $>1.5$ in $52 \%$ of DSS cases [22].

\section{Conclusion}

In our study, we conclude that commonest age of presentation of dengue fever is 5-10 yrs. Reliable diagnosis of dengue fever in endemic areas can be done by clinical parameters like presence of nausea, vomiting, pain abdomen and hepatomegaly. Ascites, pleural effusion, shock, liver dysfunction and hemorrhage are the markers for severe dengue.

Monitoring platelet count, hematocrit and WBC count are very useful for management of dengue cases, especially for judicious fluid administration and avoidance of unnecessary platelet transfusion. Low CRP was found in all the 3 groups of dengue fever, can be used to differentiate dengue fever from other diseases such as malaria, septic shock where CRP is elevated.

List of abbreviations- DF- dengue fever, DWSdengue with warning signs, SD- severe dengue, SGOT- serum glutamic oxaloacetic transaminase, SGPT- serum glutamic pyruvic transaminase, PTprothrombin time, APTT- activated partial thromboplastin time, INR- international normalized ratio, CRP- $\mathrm{C}$ reactive protein, DHF-Dengue hemorrhagic fever, DSS-Dengue shock syndrome.

Funding: Nil, Conflict of interest: None initiated, Perission from IRB: Yes

\section{Bibliography}

1. World Health Organization. Comprehensive Guidelines for Prevention and Control of Dengue and Dengue Haemorrhagic Fever, WHO, 2011: www.searo.who.int $>$ SEAROTPS60 (accessed on $8^{\text {th }}$ October 2015)

2. Hand book for Clinical Management of Dengue, WHO July 2012: www. wpro. who. int $>$ mvp $>$ documents. (accessed on 15 ${ }^{\text {th }}$ January 2017).

3. Kalayanarooj S, Vaughn DW, Nimmannitya S, Green S, Suntayakorn S, Kunentrasai N, Viramitrachai W, Ratanachu-eke S, Kiatpolpoj S, Innis BL, Rothman AL, Nisalak A, Ennis FA. Early clinical and laboratory indicators of acute dengue illness.J Infect Dis.1997Aug;176(2):313-21

4. Guzmán MG, Kourí G. Dengue diagnosis, advances and challenges. Int J Infect Dis. 2004 Mar; 8 (2):69-80.

5. Baskar C, Babu ASKK, Anandan H. Clinical Profile and Outcome of Dengue Fever in Pediatrics. 2016;5 (10):79-81.

6. Surangrat Pongpana, Apichart Wisitwong, Chamaiporn Tawichasri, Jayanton Patumanond Prognostic Indicators for Dengue Infection Severity, Int J Clin Pediatr • 2013;2(1):12-18. Doi: http://dx.doi. org/10.4021/ ijcp73w

7. Ho et al.: Clinical and laboratory predictive markers for acute dengue infection. Journal of Biomedical Science 2013; 20:75. doi:10.1186/ 1423-0127-20-75.

8. National Guidelines for Clinical Management of Dengue Fever. WHO, 2014. www.nvbdcp.gov.in (accessed on $10^{\text {th }}$ Feb 2017).

9. Jain H. Clinical profile and outcome of dengue fever in hospitalized children of South Rajasthan, India. Int J Contemp Pediatr 2016; 3(2):546-9. DOI: http: //dx.doi.org/10.18203/2349-3291.ijcp 20161035

10. Saraswathy MP, Sankari K, Sakthi Gnanavel, Sripriya Dinesh, Lakshmi priya. Incidence of dengue hemorrhagic fever in children: A report from Melmaruvathur Tamilnadu. J Pharm Sci Innov. 2013; 2 (1):34-6

11. Bhave S, Rajput C S, Bhave S. Clinical profile and outcome of dengue fever and dengue 
hemorrhagic fever in pediatric age group with special reference to WHO guidelines(2012) on fluid management of dengue fever. International Journal of Advanced Research 2015; 3 (4): 196-201.

12. Bokade C M., Chauhan U, Kamat P. Study of Hepatic Dysfunction in Dengue Fever and It's Predictor of Outcome. Int J Recent Sci Res. 2016;7 (9), pp. 13360-3.

13. Bandyopadhyay D, Chattaraj S, Hajra A, Mukhopadhyay S, Ganesan V. A Study on Spectrum of Hepatobiliary Dysfunctions and Pattern of Liver Involvement in Dengue Infection. J Clin Diagn Res. 2016 May; 10(5):OC21-6. doi: 10.7860/JCDR/2016/16946. 7784. Epub 2016 May 1.

14. Chaturvedi UC, Nagar R. Dengue and dengue haemorrhagic fever: Indian perspective.J Biosci. 2008 Nov;33(4):429-41.

15. Saha AK, Hazra SC, Chatterjee S, Maitra S. Clinico- pathological parameters predicting hemorrhage in Dengue fever in Pediatric patients of Kolkata. Transworld Medical Journal. 2013;1 (4): 116-120.

16. Gupta V, Yadav TP, Pandey RM, Singh A, Gupta M, Kanaujiya P, Sharma A, Dewan V. Risk factors of dengue shock syndrome in children. $\mathrm{J}$ Trop Pediatr. 2011 Dec;57(6):451-6. doi: 10.1093 /tropej/ fmr020. Epub 2011 Mar 2.
17. Chacko B, Subramanian G. Clinical, laboratory and radiological parameters in children with dengue fever and predictive factors for dengue shock syndrome.J TropPediatr.2008;54(2):137-140.

18. Alam K, Sulaiman SAS, Shafie AA, Yusuf E. Clinical manifestations and laboratory profile of dengue fever among the patient's general Hospital, Penang. Arch Pharma Pract. 2010;1(1):25-29

19. Karyanti MR. Clinical manifestations and hematological and serological findings in children with dengue infection. Paediatr Indones. 2011;51 (3):157-162.

20. Selvan T, Saravanan P, Nagaraj MV, Tudu MN. Study of hepatic dysfunction of dengue fever in children. Int J Contemp Pediatr 2017;4: 901-4. DOI: http: //dx.doi.org/10.18203/2349-3291.ijcp 20171695 .

21. Srinivasa S, Tanveer Nawab, Chaithanya C Nair. Clinical profile and ultasonogaphic findings in children with dengue fever. Curr Pediatr Res 2014; 18 (2): 87-90

22. Jagadishkumar $\mathrm{K}$, Jain $\mathrm{P}$, Manjunath $\mathrm{VG}$, Umesh L. Hepatic involvement in dengue Fever in children.Iran J Pediatr. 2012 Jun;22(2):231-6.

23. Selvan T, Purushotham D R, Swamy N, Giridhar, Kumar M, Suresh et al. study of prevalence and hepatic dysfunction in dengue fever in children.Sch.J.App.Med.Sci.,2015;3(5D):2071-4.

\section{How to cite this article?}

Prabhavathi R, Madhusudan S R, Suman M.G, Govindaraj M, Puttaswamy M. Study of clinical and laboratory predictive markers of dengue fever and severe dengue in children. J PediatrRes.2017;4(06):397404.doi:10.17511/ijpr.2017.i06.08. 